\title{
Decisiones de diversificación y spin off. Ciclos de expansión y ciclos de enfoque: Analisis retrospectivo de las evidencias del caso Bavaria
}

\author{
Hernán Herrera-Echeverri*
}

Fecha de recibido: 4 de abril 2018 Fecha de aprobado: 16 de julio de 2018

Para citar: Herrera-Echeverri, H. (2019). Decisión de diversificación y decisión de spin off. Ciclos de expansión y ciclos de enfoque: Análisis retrospectivo de las evidencias del caso Bavaria. Universidad \& Empresa, 21(36), 228-260. DOI: http://dx.doi.org/10.12804/revistas.urosario.edu.co/empresa/a.6649 


\section{Resumen}

Esta propuesta utiliza el caso del Antigüo Grupo Empresarial Bavaria para analizar tres aspectos relacionados con la diversificación no relacionada y el proceso de spin off de las firmas. Primero se establece que las empresas con unidades de negocio caracterizadas por excesos de liquidez, se diversifican de manera no relacionada, debido a que la existencia de un mercado interno de capitales les permite derribar sus restricciones de financiación e incrementar la rentabilidad corporativa. Segundo, se demuestra que la decisión de diversificación de un gerente corporativo es inversamente proporcional a la magnitud del costo esperado por la dilución de esfuerzo del gerente de la nueva unidad de negocio. Finalmente, se muestra cómo la diversificación no relacionada presenta un límite en su contribución al incremento de la rentabilidad corporativa, en cuyo caso se puede inducir la decisión de realizar un spin off para delimitar, especializar y mejorar la calidad de las decisiones de asignación de recursos. Se concluye que lo anterior puede explicar por qué en algunos grupos económicos se observan ciclos de diversificación y ciclos de enfoque. Cuando el grupo económico desea incrementar la rentabilidad inicia su proceso de diversificación; una vez llega al umbral que hace que la probabilidad de acierto en sus decisiones de inversión se reduzca, empieza nuevamente su proceso de enfoque, realizando separación de sus unidades no relacionadas. Este ciclo se puede repetir en el desarrollo histórico de un grupo empresarial.

Palabras clave: grupos empresariales, diversificación no relacionada, escisiones, mercados internos de capital.

\section{Diversification Decision and Spin-Off Decision. Expansion Cycles and Focus Cycles: Retrospective Analysis of Evidence in the Bavaria Case Abstract}

This proposal uses the case of Grupo Empresarial Bavaria to analyze three aspects of the unrelated diversification and the spin off process. First, it is established that firms with business units characterized by cash excess, start unrelated diversification processes, because internal capital market allows them to bring down their funding constraints and increase corporate profitability. Second, we demonstrate that the diversification decision of a corporate manager is inversely proportional to the magnitude of the expected cost by the effort dilution of the manager of the new business unit. And finally, it is shown that unrelated diversification has a limit on their contribution to increased corporate profitability, in which case it may induce the decision to spin off for delimit, specialize and improve the quality of allocation decisions resources. This explains why some corporative groups present focus cycles and diversification cycles. When the economic group wants to increase the profitability starts a diversification process, once the threshold is overtaken, the probability of success in their investment decisions is reduced, then the focus process begins again by separating unrelated units. This cycle can be repeated in the historical development of a business group.

Keywords: Business groups, unrelated diversification, scission, internal capital markets.

\section{Decisão de diversificação e decisão de Spin-off. Ciclos de expansão e ciclos de enfoque: Análise retrospetiva das evidências do caso Bavaria Resumo}

Esta proposta utiliza o caso do Antigo Grupo Empresarial Bavaria para analisar três aspetos relacionados com a diversificação não relacionada e o processo de spin off das empresas. Primeiro se estabelece que as empresas com unidades de negócio caracterizadas por excessos de liquidez, se diversificam de maneira não relacionada, devido a que a existência de um mercado interno de capitais, lhes permite derribar suas restrições de financiamento e incrementar a rentabilidade corporativa. Segundo, se demonstra que a decisão de diversificação de um gerente corporativo é inversamente proporcional a magnitude do custo esperado pela diluição de esforço do gerente da nova unidade de negócio. E finalmente, se mostra como a diversificação não relacionada apresenta um limite em sua contribuição ao incremento da rentabilidade corporativa, em cujo caso se pode induzir a decisão de realizar um spin off, para delimitar, especializar e melhorar a qualidade das decisões de assinação de recursos. Conclui-se que o anterior pode explicar porque em alguns grupos econômicos se observam ciclos de diversificação e ciclos de enfoque. Quando o grupo econômico deseja incrementar a rentabilidade inicia seu processo de diversificação, uma vez chega ao umbral que faz que a probabilidade de acerto em suas decisões de investimento se reduza, então começa novamente seu processo de enfoque, realizando separação de suas unidades não relacionadas. Este ciclo se pode repetir no desenvolvimento histórico de um grupo empresarial.

Palavras-chave: grupos empresariais, diversificação não relacionada, cisões, mercados internos de capital. 


\section{Introducción}

Muchos argumentos se han desarrollado en contra de la diversificación no relacionada. ${ }^{1}$ Costos por errores potenciales en la localización de recursos (Meyers, Milgron \& Roberts, 1992; Ramaswamy, Purkayastha \& Petitt, 2017), mecanismos más costosos para controlar el desempeño y sistemas de recompensa de los gerentes de unidades de negocio en contra del desempeño corporativo (Hermalin \& Weisbach, 1998. Hoechle, Schmid, Walter \& Yermack, 2012), distorsiones en la localización de recursos entre divisiones por pugnas internas de poder (Rajan, Servaes, \& Zingales, 2000), disminución en los precios de las acciones por anuncio de diversificación en las firmas (Manne, 1965), los accionistas de firmas que llevan a cabo adquisición de otras firmas para diversificarse de manera no relacionada, no se benefician de esas adquisiciones (Morck, Schleifer \& Vishny, 1990), las acciones de las firmas no diversificadas se tranzan con un descuento frente a las firmas no diversificadas de sectores similares (Lang \& Stulz, 1994; Berger \& Ofec, 1995; Mansi \& Reeb, 2002).

No obstante todas las advertencias anteriores, existen muchas corporaciones que deciden emprender diversificaciones no relacionadas en su plan de desarrollo de negocios. Las razones para justificar tal nivel de diversificación no relacionada pueden ser varias: la búsqueda de economías de escala y de alcance (Brush, 1996; Weston, 1970; Chandler, 1977), economizar costos de transacción (Teece, 1982), para que los gerentes puedan reducir el riesgo idiosincrático y capturar mayores beneficios privados (Aggarwal \& Samwicic, 2003; Amihud \& Lev, 1981), para hacer más fácil la reasignación de activos entre unidades de negocio (Gertner, Scharfstein \& Stein, 1994), la diversificación le permite al gerente crear una gran corporación (Jensen, 1986) y hacerse más indispensable para manejarla (Shleifer \& Vishny, 1989), y para incrementar la capacidad de endeudamiento y los escudos fiscales por diversificación del riesgo operativo (Lewellen, 1971; Khanna \& Palepu, 2000; Lins \& Servaes, 2002; Zheng, 2017).

Este artículo se aparta de las anteriores justificaciones y hace un analisis retrospectivo, antes de ser adquirido por Sab-Miller, del Grupo Empresarial Bavaria, para plantear

\footnotetext{
Se utiliza indistintamente el término diversificación y diversificación no relacionada para referirse a corporaciones que han hecho inversiones en empresas con unidades de negocio que difieren en su objeto de negocio, pero que son controladas por decisiones que se toman desde una gerencia corporativa.
} 
respuestas adicionales a dos preguntas relacionadas con la diversificación no relacionada y el proceso de spin off de las firmas. ¿Por qué una empresa decide combinar distintas unidades de negocio no relacionadas bajo una administración corporativa?, ¿por qué una firma que ha hecho previamente diversificación no relacionada, decide que debe hacer un spin off de una determinada unidad de negocio? Las respuestas a estas dos preguntas son desarrolladas a partir del planteamiento de tres proposiciones teóricas. La primera se fundamenta en que las empresas con unidades de negocio caracterizadas por excesos de liquidez, se diversifican de manera no relacionada, porque la existencia de un mercado interno de capital permite derribar las restricciones de financiación para incrementar su rentabilidad corporativa, lo que se consigue porque se invierte en la creación de nuevas unidades de negocio con alto retorno, aunque no tengan alta liquidez. ${ }^{2}$ La segunda proposición aborda el problema del factor de dilución de esfuerzo (Grossman \& Hart, 1986; Stein, 1997; Scharfstein \& Stein, 2000.). Este documento contribuye al tema demostrando que entre menor sea la magnitud de la caída de los flujos de caja por el efecto de la dilución de esfuerzo del gerente de la unidad de negocio, mayor será la disposición a diversificar y mayor será el beneficio privado que el gerente corporativo está dispuesto a ceder al gerente de la unidad de negocio.

La tercera proposición establece en qué momento la empresa matriz decide hacer un spin off de una unidad de negocio no relacionada, con el fin de concentrarse y especializar los negocios para incrementar la rentabilidad. Muchos estudios han mostrado evidencias acerca de cómo los spin off crean valor (Miles \& Rosenfield, 1983; Kudla \& Mclnish, 1983; Hite \& Owers, 1983; Schipper \& Smith, 1983), en este artículo se relaciona esa creación de valor con la estrategia de enfocar el objeto de negocio de la corporación. La intuición utilizada es que en la medida que la diversificación corporativa se incrementa, surgen problemas de asignación adecuada de recursos y el spin off puede ser considerado como una estrategia para incrementar la eficiencia en la asignación de capital y disminuir la dilución de esfuerzo gerencial (Ahn \& Denis, 2004; Scharfstein \& Stein, 2000; Rajan et al., 2000; Gertner, Powers \& Scharfstein, 2002). El caso del Grupo Económico Bavaria es utilizado para ilustrar las conclusiones propuestas, porque su desarrollo histórico denota

Este planteamiento es afín a las ideas centrales de Stein (1997), Lamont (1997), Shin y Stulz (1998) acerca de las principales ventajas de la existencia de mercados internos de capital en una corporación, y a Williamson (1975), quien argumenta que los capitales internos de capital son más eficientes, porque es más probable que los gerentes corporativos estén mejor informados acerca de las buenas oportunidades de inversión. 
los aspectos considerados en las proposiciones. El artículo sigue de la siguiente manera: En la primera parte se demuestran las proposiciones enunciadas mediante el uso de un modelo teórico; en la segunda se hace una descripción del desarrollo histórico del Grupo Empresarial Bavaria, centrándonos en los aspectos de relevancia para el desarrollo de las conclusiones del artículo y en la parte final, se exponen las conclusiones.

\section{Análisis teórico}

\subsection{Incremento de la rentabilidad a través de la diversificación y el uso de los mercados internos de capital}

Un gerente corporativo tiene incentivos para controlar un mayor monto de fondos de capital, con el fin de obtener un mayor beneficio privado (Gertner, Scharfstein \& Stein, 1994) por tanto, también deseará invertir esos fondos de capital en nuevas unidades de negocio, que le representen oportunidades de obtener una rentabilidad corporativa mayor y mejorar su posición de poder. Cuando las oportunidades de ganar mayor rentabilidad se presenten a través de unidades de negocio no relacionadas, el gerente corporativo tendrá poca experiencia en el manejo de la nueva unidad de negocio, y deberá contratar un gerente experto para dicha unidad (Campa \& Kedia, 2002).

Se pueden presentar dos casos; en el primero, la corporación no tiene unidades de negocio que le provean flujos de caja estables y debe buscar financiación externa para invertir en nuevas unidades de negocio. En el segundo, la corporación posee unidades de negocio que le proveen flujos de caja estables, y el gerente preferirá invertirlos en las nuevas unidades de negocio, en vez de optar por devolverlos a los dueños de la empresa en forma de dividendos (Stein, 2003). 


\subsubsection{La existencia de restricciones de financiación por proveedores externos de capital}

El retorno mínimo requerido por un inversionista es $R_{i}=1+r_{i}$ (uno más la tasa de retorno mínina requerida por el inversionista) y $R_{c}$ el retorno requerido por la corporación para que una unidad de negocio incremente la rentabilidad total de la corporación $\left(R_{c}>1+r_{c}\right.$ :el retorno requerido debe ser mayor a uno más la tasa de retorno actual de la corporación). La corporación puede invertir $I_{1}$ (inversión baja) o $I_{2}$ (inversión alta; $2 I_{1}=I_{2}$ ). El retorno de la inversión depende del estado de la naturaleza que puede ser bueno $(B)$ o malo $(M)$. Los flujos de caja que resultan en cada estado de la naturaleza se muestran en la tabla 1:

Tabla 1. Niveles de inversión, valor presente de los flujos de retorno de la inversión y beneficios privados de los gerentes, dependiendo de los estados de la naturaleza

\begin{tabular}{llll}
\hline $\begin{array}{c}\text { Estado de } \\
\text { la naturaleza }\end{array}$ & \multicolumn{1}{c}{$\begin{array}{c}\text { Nivel de } \\
\text { inversión }\end{array}$} & $\begin{array}{c}\text { Valor presente flujo } \\
\text { de caja de retorno }\end{array}$ & $\begin{array}{c}\text { Beneficios privados } \\
\text { de los gerentes }\end{array}$ \\
\hline \multirow{2}{*}{ Malo } & $I_{1}$ & $Y_{1}$ & $f Y_{1}$ \\
\cline { 2 - 4 } & $I_{2}$ & $Y_{2}$ & $f Y_{2}$ \\
\hline \multirow{2}{*}{ Bueno } & $I_{1}$ & $\theta_{1} Y_{1}$ & $f \theta_{1} Y_{1}$ \\
\cline { 2 - 4 } & $I_{2}$ & $\theta_{2} Y_{2}$ & $f \theta_{2} Y_{2}$ \\
\hline
\end{tabular}

Fuente: elaboración propia.

Se asume que:

- $Y_{1,} Y_{2}$ son los valores presentes de los flujos de caja resultantes de cada nivel de inversión, verificables a un costo determinado.

- En el estado malo de la naturaleza $I_{1}<Y_{1}<Y_{2}<I_{2}$; por tanto, si se sabe que el estado de la naturaleza es malo, la mejor inversión es $I_{l}$.

- $\theta_{2}>\theta_{1}>1$ son los factores de incremento de los valores presentes de los flujos de caja si el estado de la naturaleza es bueno y se hace la inversión baja o alta, respectivamente. Este supuesto implica que así como cuando las cosas no funcionan bien, las perdidas pueden ser mayores en la medida que se invierte más (por eso $Y_{2}<I_{2}$, en el estado malo de la naturaleza), también cuando las cosas salen bien los retornos pueden ser superiores si hace una inversión alta (por eso $\theta_{2}>\theta_{1}$ ). 
- Cuando el estado de la naturaleza es bueno $\theta_{2} Y_{2} / I_{2}>R_{c}>\theta_{1} Y_{1} / I_{1}>R_{i}$. Por tanto, si se sabe que el estado de la naturaleza es bueno, la mejor inversión es $I_{2}$.

- La probabilidad ex-ante de que el estado bueno ocurra es $p$ y la probabilidad de que ocurra el estado malo es $1-p$.

El gerente de la nueva unidad estratégica de negocio observa directamente el estado de la naturaleza, pero el gerente corporativo, los inversionistas y los proveedores de financiación, no. Los proveedores de financiación y los inversionistas solo estarían de acuerdo con realizar una inversión acorde con el estado de la naturaleza; sin embargo, los gerentes corporativos y los de las unidades de negocios tienen la tendencia de realizar reportes con sobreestimación de los posibles resultados de la inversión, debido a que ellos derivan beneficios privados por el incremento de los recursos bajo su control. El parámetro $f<1$ se utiliza para indicar la porción de los valores presentes de los flujos de caja que gerentes corporativos obtienen como beneficio privado.

Para modelar esta tendencia a la sobreestimación de la gerencia, suponemos que los beneficios privados (no observables) de los gerentes son proporcionales al monto de los flujos de caja resultantes de las inversiones realizadas. El beneficio privado del gerente se puede calcular como el producto entre el flujo de caja obtenido en cada estado de la naturaleza, dependiendo del monto de la inversión realizada, por el parámetro $f$.

Cuando se busca financiación externa, los proveedores de capital tienen la opción de financiar $I_{1} O I_{2}$. Las utilidades obtenidas en cada estado de la naturaleza, dependiendo del nivel de inversión realizada se muestran en la figura 1.

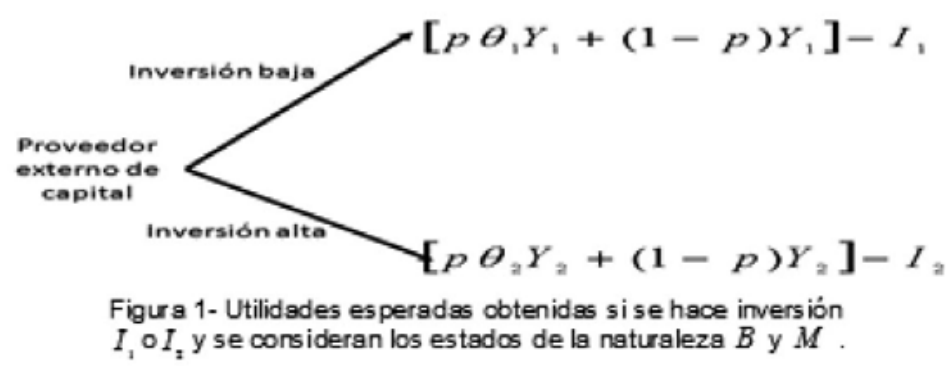

Figura 1. Utilidades esperadas obtenidas si se hace inversión $I_{1} \circ I_{2}$ y se consideran los estados de la naturaleza B y M

Fuente: elaboración propia. 
El valor esperado de la utilidad cuando se hace la inversión baja es mayor que cero, porque

$$
\left[p \theta_{1} Y_{1}+(1-p) Y_{1}\right]>Y_{1}>I_{1}
$$

De esta manera, la estrategia dominante para un inversionista sería asegurarse obteniendo un retorno positivo sin importar el estado de la naturaleza, además porque si el criterio de aceptación de los proveedores de capital es su retorno mínimo requerido, siempre se cumple que:

$$
\frac{\left[p \theta_{1} Y_{1}+(1-p) Y_{1}\right]}{I_{1}}>\frac{Y_{1}}{I_{1}}>R_{i}
$$

Sin embargo, dado que $R_{i}<R_{c}$, el gerente corporativo no estaría satisfecho, pues la rentabilidad corporativa estaría disminuyéndose si se hace la inversión baja. En el caso que se haga la inversión alta, la utilidad esperada sería:

$$
u=\left[p \theta_{2} Y_{2}+(1-p) Y_{2}\right]-I_{2}
$$

Pero $\operatorname{comol}_{2}<I_{2}$, existe un nivel de $p$ que puede hacer que la utilidad sea negativa para los inversionistas. Este nivel sería: $P<\frac{I_{2}-Y_{2}}{Y_{2}\left(\theta_{2}-1\right)}$

Es decir, los proveedores de capital evitarán hacer la inversión alta cuando la creencia ex-ante de que el estado de la naturaleza va a ser bueno, es menor al cociente entre el monto de la pérdida posible y la multiplicación del flujo esperado por el retorno menos uno, es decir, los proveedores externos de capital evitarán financiar inversiones altas ante valores pequeños de $p$. Luego, las empresas que deben acceder a recursos externos para financiar nuevas unidades de negocio pueden enfrentar problemas para conseguir los fondos totales para la expansión, mientras que empresas que disponen de otras unidades de negocio que proveen liquidez interna, pueden evitar esta restricción de capital. 


\subsubsection{El incremento de la rentabilidad mediante la diversificación cuando se utilizan los mercados internos de capital para superar las restricciones de capital}

Si un gerente corporativo toma la decisión de diversificar debe contratar un gerente para la nueva unidad de negocio. Varios autores (Grossman \& Hart, 1986; Stein, 1997; Scharfstein \& Stein, 2000) mencionan que la existencia de una gerencia corporativa representa un costo a causa de la dilución de esfuerzos que provoca, pues la fracción de beneficios privados que obtiene la gerencia corporativa se obtiene de la disminución del beneficio del gerente de la unidad de negocio. El gerente de la unidad de negocio puede reducir su esfuerzo, debido a que se siente menos motivado por la disminución de su beneficio privado a causa de la existencia de la gerencia corporativa. Stein (1997) propone capturar esta dilución del esfuerzo suponiendo que cuando una unidad de negocio es supervisada por una gerencia corporativa, los flujos de caja obtenidos caen en una cifra determinada por un factor $k<1$.

Otro elemento que caracteriza al gerente corporativo es que cuando tiene la posibilidad de suplir las restricciones de financiación mediante el uso de los mercados internos de capital, puede redistribuir los fondos, retirándolos de unidades de negocio con bajo retorno y asignándolos a las unidades de negocio con mayor rentabilidad. Se puede demostrar que la rentabilidad corporativa incrementa cuando existe un mercado interno de capital y la posibilidad de reasignación de recursos por el gerente corporativo, probando que la utilidad esperada en este caso es mayor a la utilidad esperada que se obtiene cuando no existen mercados internos de capital.

- Utilidad esperada cuando no existe la corporación y cada unidad de negocio intenta buscar por su cuenta fondos externos:

$U_{f}=2\left(\left[p \theta_{1} Y_{1}+(1-p) Y_{1}\right]-I_{1}\right)$

En este caso solo se logra la utilidad asociada a la inversión baja que se consigue del mercado. 
- Utilidad esperada cuando existe un gerente corporativo con fondos internos limitados y la posibilidad de reasignar recursos:

$$
U_{i}=\left[2 p^{2} \theta_{1} Y_{1} k+2(1-p)^{2} Y_{1} k+2(1-p) p Y_{2} \theta_{2} k\right]-2 I_{1} \text {, }
$$

El factor de dilución $K$ el cual puede hacer que $U_{f}$ sea mayor que $U_{i}$. No obstante cuando los proyectos estén en diferentes estados, los cual ocurre con una probabilidad $2(1-p) p$, el proyecto que está en estado bueno recibe todos los recursos y el proyecto que está en el estado malo no recibe recursos. Esta situación hace que se pueda compensar la presencia de $K$ y que $U_{f}$ sea menor que $U_{i}$.

- Utilidad esperada cuando hay fondos internos de capital y no hay restricciones de financiación

$$
\begin{aligned}
& U_{n}=2 p^{2}\left[\theta_{2} Y_{2} k-I_{2}\right]+2(1-p)^{2}\left[Y_{1} k-I_{1}\right] \\
& +2(1-p) p\left[Y_{1} k-I_{1}+\theta_{2} Y_{2} k-I_{2}\right]
\end{aligned}
$$

Como las unidades de negocio en estado malo tienen una utilidad positiva si la inversión es baja, la estrategia dominante para el gerente es continuar invirtiendo en ellas, y además hacer la inversión alta en las unidades de negocio que están en el estado bueno, porque así se logra una $U_{n}$ que es mayor que $U_{i}$.

Proposición 1. Un gerente corporativo con acceso a mercados internos de capital proporcionados por flujos de caja de unidades de negocio con alta liquidez utilizará dichos mercados internos de capital, para superar las restricciones de financiamiento e incrementará la inversión en otras unidades de negocio, con el fin de lograr mayores niveles de rentabilidad corporativa.

Prueba: la anterior proposición queda demostrada con lo realizado en los literales 2.1. y 2.2. En el literal 2.1. se demostró que el acceso a mercados internos de capital es una forma de evitar las restricciones de capital. En el literal 2.2. se demostró cómo un gerente corporativo con acceso a mercados internos de capital proporcionados por flujos de caja de unidades de negocio con alta liquidez, puede incrementar la inversión en otras unidades 
de negocio, con el fin de lograr mayores niveles de rentabilidad corporativa, quedando entonces demostrada la proposición.

\subsection{Relación entre beneficios privados del gerente corporativo y diversificación}

Un gerente corporativo aporta su habilidad para supervisar y evaluar información ex-ante acerca de las posibles unidades de negocio a impulsar, pero no dispone de recursos propios para invertir. A los gerentes corporativos les son delegados derechos de control por parte de los dueños de la empresa (Hart \& Moore, 1990) que les permiten capturar una fracción $f<1$ de los flujos de caja, que obtienen las diferentes unidades de negocio bajo su supervisión. Cuando un gerente corporativo decide diversificar y contrata a un gerente de unidad de negocio, le otorga una fracción $\phi<1$ del beneficio privado obtenido en esa unidad de negocio. Podemos reconstruir la tabla de valores presentes de los flujos de caja de la inversión, quedando tal como se muestra en la tabla 2.

Tabla 2. Niveles de inversión, valor presente de los flujos de retorno de la inversión y beneficios privados de los gerentes corporativos y de los gerentes de unidades de negocio, dependiendo de los estados de la naturaleza y del factor de dilución del esfuerzo

\begin{tabular}{ccccc}
\hline $\begin{array}{c}\text { Estado de la } \\
\text { naturaleza }\end{array}$ & $\begin{array}{c}\text { Nivel de } \\
\text { inversión }\end{array}$ & $\begin{array}{c}\text { Valor presente } \\
\text { flujo de caja de } \\
\text { retorno }\end{array}$ & $\begin{array}{c}\text { Beneficios privados } \\
\text { de los gerentes de } \\
\text { unidades de negocios }\end{array}$ & $\begin{array}{c}\text { Beneficios privados } \\
\text { de los gerentes } \\
\text { corporativos }\end{array}$ \\
\hline $\begin{array}{c}\text { Malo } \\
\text { Probalidad } \\
\text { 1-P }\end{array}$ & $I_{1}$ & $Y_{1}$ & $\phi K f Y_{1}$ & $(1-\phi) \mathscr{K} f Y_{1}$ \\
\cline { 2 - 5 } $\begin{array}{c}\text { Bueno } \\
\text { Probalidad } \\
P\end{array}$ & $I_{2}$ & $Y_{2}$ & $\phi K f Y_{2}$ & $(1-\phi) K f Y_{2}$ \\
\cline { 2 - 5 } & $I_{1}$ & $\theta_{1} Y_{1}$ & $\phi K f \theta_{1} Y_{1}$ & $(1-\phi) K f \theta_{1} Y_{1}$ \\
\hline$I_{2}$ & $\theta_{2} Y_{2}$ & $\phi K f \theta_{2} Y_{2}$ & $(1-\phi) K f \theta_{2} Y_{2}$ \\
\hline
\end{tabular}

Fuente: elaboración propia.

Con esta estructura de información, el gerente corporativo enfrenta una tensión que está determinada por su necesidad de incrementar la rentabilidad corporativa, abriendo nuevas unidades de negocio, pero al mismo tiempo debe controlar que el beneficio privado y la dilución del esfuerzo del gerente de la unidad de negocio no haga que la apertura tenga un retorno negativo y termine disminuyendo la rentabilidad corporativa. 
Proposición 2. Entre menor sea la magnitud de la caída de los flujos de caja por el efecto de la dilución de esfuerzos del gerente de la unidad de negocio ante la presencia del gerente corporativo, mayor será el beneficio privado que el gerente corporativo está dispuesto a ceder al gerente de la unidad de negocio.

Prueba: la gerencia corporativa nombra los nuevos gerentes cuando el beneficio privado inicial del gerente corporativo, antes de abrir la nueva unidad de negocio, sea menor al beneficio privado que recibiría proveniente de la diversificación, en cuyo caso nombraría a un gerente para cada unidad de negocio. Lo anterior se puede expresar de la siguiente manera:

$$
\begin{aligned}
& \left(2 p^{2}\left[\theta_{2} Y_{2} k\right]+2(1-p)^{2}\left[Y_{1} k\right]+2(1-p) p\left[Y_{1} k+\theta_{2} Y_{2} k\right]\right) f(1-\phi) \\
& {\left[p \theta_{2} Y_{2}+(1-p) Y_{1}\right] f}
\end{aligned}
$$

La ecuación (7) representa el beneficio privado para el gerente corporativo si decide diversificar y la ecuación (8) representa el beneficio privado para el gerente corporativo si no diversifica. Para que haya diversificación $(7)>(8)$, es decir

$$
2 k\left(p^{2}\left[\theta_{2} Y_{2}\right]+(1-p)^{2}\left[Y_{1}\right]+(1-p) p\left[Y_{1}+\theta_{2} Y_{2}\right]\right)(1-\phi)>p \theta_{2} Y_{2}+(1-p) Y_{1}
$$

Si se expresa el término del que está entre paréntesis en el lado izquierdo de la desigualdad como el ingreso esperado por la diversificación, $E[d]$ y el término de la derecha en ingreso esperado si no hay diversificación, $E[n]$, es decir:

$$
\begin{aligned}
& E[d]=p^{2}\left[\theta_{2} Y_{2}\right]+(1-p)^{2}\left[Y_{1}\right]+(1-p) p\left[Y_{1}+\theta_{2} Y_{2}\right] \\
& E[n]=p \theta_{2} Y_{2}+(1-p) Y_{1}
\end{aligned}
$$

Obtenemos que:

$$
\phi<1-\frac{E[n]}{2 k E[d]}
$$

Se concluye que entre menor sea la magnitud de la caída de los flujos de caja por el efecto de la dilución de esfuerzo del gerente de la unidad de negocio, mayor será el beneficio privado que el gerente corporativo este dispuesto a ceder al gerente de la unidad de negocio, quedando probada la proposición. Esto es complementario a lo planteado en otros 
estudios acerca de los incentivos de los gerentes corporativos para tomar decisiones de diversificación (Jensen, 1986; Stulz, 1990; Jensen \& Murphy, 1990). Si la dilución de esfuerzo está en función de la contribución de la gerencia corporativa al desempeño de la unidad de negocio, habrá más dilución de esfuerzo cuando el gerente de la unidad de negocio siente que la gerencia corporativa no aporta nada a su trabajo, pero sí extrae beneficios. Como el aporte de la gerencia corporativa es menor cuando existe alta diversificación, se puede expresar la dilución de esfuerzos $(K(n))$, en función decreciente del número de unidades de negocio diferentes dentro de la corporación $(n)$ :

$$
K(n)=k ; \frac{\partial K(n)}{\partial n}<0
$$

Lo anterior implica que un gerente de unidad de negocio no realice el esfuerzo adecuado, si considera que su unidad es de alta especificidad y que la gerencia corporativa asigna los recursos sin conocer, ni aportar en las decisiones para desarrollar dicha la unidad de negocio, es decir, cuando los niveles de diversificación son altos.

\subsection{Limites al alcance de la diversificación no relacionada y decisión de spin off}

El lanzamiento de nuevas unidades de negocio puede implicar sacrificios en competitividad de las actuales unidades de negocio, fenómeno que se conoce como la pérdida de foco de la empresa. Al margen de que un spin off puede constituir un incentivo para mejorar el desempeño de los gerentes de las unidades de negocio, por la desaparición del fenómeno de dilución de esfuerzo (Aron, 1991), algunos grupos económicos que optaron inicialmente por diversificarse, posteriormente realizaron un spin off con el fin de concentrar cada empresa separada en su negocio central; la corporación a través del spin off busca crear personas jurídicas diferentes para propiciar un mejor manejo de actividades productivas distintas. En este parte del caso se muestra que una de las posibles causas que induce a la realización del spin off es porque al delimitar y especializar el trabajo del grupo gerencial, se mejora la calidad de las decisiones de inversión.

Para demostrar lo anterior se comparan las probabilidades de éxito en la toma de decisiones de inversión de un gerente corporativo cuando está en una empresa enfocada y cuando está en una empresa diversificada. ¿Hasta qué punto pueden mantenerse niveles adecuados de calidad de la gestión que ejerce una gerencia corporativa en una empresa 
diversificada? Para evitar que errores en la toma de decisiones de asignación de recursos erosionen el incremento de las utilidades que pudieron haber surgido de la diversificación. En nuestro análisis consideramos que la decisión de invertir en una unidad de negocio está determinada por la siguiente secuencia que se expone en la figura 2

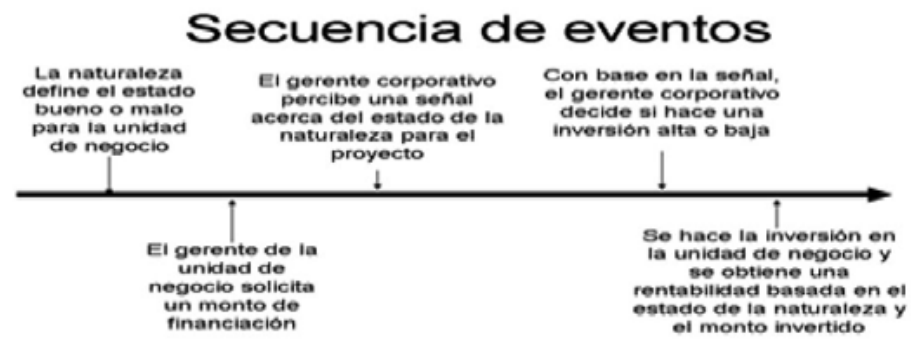

Figura 2. Secuencia de eventos que sigue la toma de una decisión de invertir en una unidad de negocio

Fuente: elaboración propia.

El trabajo del gerente corporativo es evaluar el merito de cada unidad de negocio para invertir en ella, inicialmente con base en la solicitud del gerente de la unidad de negocio. Sin embargo, como ya se ha dicho, el gerente de la unidad de negocio tiene tendencia a querer dominar la mayor cantidad de recursos posibles, por ello el gerente corporativo, además de escuchar la solicitud, se basa en un conjunto de información que conforma una creencia acerca de si el estado de la naturaleza le es o no favorable a una determinada unidad de negocio. Así, si suponemos que existen dos unidades de negocio $(i, j)$ en una corporación, el gerente corporativo acierta cuando hace una inversión alta $\left(L=I_{2}\right)$ en una unidad de negocio que enfrentará un estado bueno de la naturaleza $(B)$ o cuando hace una inversión baja ( $C=I_{1}$ ) en una unidad de negocio que enfrenta un estado malo $(M)$ de la naturaleza; esta probabilidad de acierto la denotaremos como $q$, y puede expresarse de la siguiente manera:

$$
\mathrm{P}\left(\mathrm{C}_{\mathrm{i}} / \mathrm{M}_{\mathrm{i}}\right)=\mathrm{P}\left(\mathrm{L}_{\mathrm{i}} / \mathrm{B}_{\mathrm{i}}\right)=\mathrm{q} \text {. }
$$

El caso contrario indica que la decisión fue un desacierto y la probabilidad de ocurrencia será denotada como:

$$
\mathrm{P}\left(\mathrm{C}_{\mathrm{i}} / \mathrm{B}_{\mathrm{i}}\right)=\mathrm{P}\left(\mathrm{L}_{\mathrm{i}} / \mathrm{M}_{\mathrm{i}}\right)=1-\mathrm{q}
$$

La rentabilidad de la corporación en un determinado periodo está basada en el juicio del gerente corporativo, quien toma como base sus creencias acerca del estado de la naturaleza, 
para una determinada unidad de negocio y decide el monto de la inversión. El nivel de correlación entre estos errores de juicio sobre los niveles de inversión está determinado por el nivel de diversificación entre las unidades de negocio, de tal forma que si existe foco, el error cometido al determinar el nivel de inversión en una unidad de negocio se propagará a las otras con su mismo objeto de negocio. Para incluir la argumentación anterior en el modelo diremos que: ${ }^{3}$

$$
\mathrm{P}\left(\mathrm{C}_{\mathrm{i}} / \mathrm{B}_{\mathrm{i}}, \mathrm{B}_{\mathrm{j}}, C_{\mathrm{j}}\right)=\mathrm{P}\left(\mathrm{C}_{\mathrm{i}} / \mathrm{M}_{\mathrm{i}}, \mathrm{M}_{\mathrm{j}}, L_{\mathrm{j}}\right)=(1-\mathrm{q}) *(1+\alpha)
$$

Donde $\alpha$ es un parámetro entre cero y uno que mide el grado de foco en la corporación, entre más alto $\alpha$ más enfocada es la corporación. Para simplificar el desarrollo del modelo, seguiremos a Stein (1997), quien asume que cuando la gerencia corporativa toma una mala decisión en una unidad de negocio, esto no mejora la probabilidad de que se acierte en otra. Por tanto dejaremos:

$$
\mathrm{P}\left(\mathrm{C}_{\mathrm{i}} / \mathrm{M}_{\mathrm{i}}, B_{\mathrm{j}}, C_{\mathrm{j}}\right)=\mathrm{P}\left(\mathrm{L}_{\mathrm{i}} / \mathrm{B}_{\mathrm{i}}, M_{\mathrm{j}}, L_{\mathrm{j}}\right)=q
$$

Debido al conocimiento que el gerente corporativo tiene de las antiguas unidades de negocio que están altamente relacionadas, entonces q estará entre 1 y 0.5 . (1>q>0.5).

Cuando existe diversificación, el objeto de negocio de las unidades $i$ y $j$ es diferente; por consiguiente, el error cometido al determinar el nivel de inversión en la unidad de negocio $i$ es poco probable que se propague a la unidad de negocio $j$. Además, las probabilidades de que la gerencia acierte en la decisión de inversión en ambas unidades es diferente, por tanto asumiremos que la probabilidad de acertar en la unidad de negocio $i$ sigue siendo $q$, mientras que la probabilidad de acertar en el la decisión de inversión en la unidad de negocio $j$ es $r$ :

$$
P\left(C_{i} / M_{i}\right)=P\left(L_{i} / B_{i}\right)=q \quad ; \quad P\left(C_{j} / M_{j}\right)=P\left(L_{j} / B_{j}\right)=r \quad ; \quad P\left(L_{i} / M_{i}\right)=P\left(C_{i} / B_{i}\right)=1-q \quad ; \quad P\left(L_{j} / M_{j}\right)=P\left(C_{j} / B_{j}\right)=1-r
$$

$3 \quad \mathbf{P}\left(\mathbf{C}_{\mathrm{i}} / \mathbf{B}_{\mathrm{i}}, \mathbf{B}_{\mathrm{j}}, C_{\mathrm{j}}\right)$ puede leerse como la probabilidad de que el gerente corporativo decida hacer una inversión baja en la unidad de negocio i, dado que el estado de la naturaleza para i es bueno, y que realizó una inversión baja en la unidad de negocio j, dado que el estado de la naturaleza para j era bueno, es decir, es la probabilidad de que se equivoque en la decisión de inversión en la unidad de negocio i, dado que se equivocó en la unidad de negocio j, cuando el estado de la naturaleza es bueno. Una interpretación similar se puede hacer para $\mathbf{P}\left(\mathbf{C}_{\mathrm{i}} / \mathbf{M}_{\mathrm{i}}, \mathbf{M}_{\mathrm{j}}, L_{\mathrm{j}}\right)$ cuando el estado de la naturaleza es malo. 
Si la corporación antes solo maneja unidades de negocio del tipo de negocio $i$ y posteriormente se diversificó, invirtiendo en unidades de negocio del tipo $j$, entonces $q>r$, porque la gerencia corporativa tiene más afinadas las creencias sobre $i$ que sobre $j$. En la medida que se incrementa la diversificación, se reduce la probabilidad de acierto en las decisiones de inversión, de tal forma que la probabilidad de acierto en las decisiones de inversión de la gerencia es una función decreciente del nivel de diversificación de las unidades de negocio Esta conclusión es consistente con lo planteado por otros autores Stein (1997), Ahn y Denis (2004), Scharfstein y Stein (2000), Rajan et al. (2000), y Gertner, Powers y Scharfstein (2002). Un resumen acerca del conjunto de probabilidades se muestra en la tabla 3.

Tabla 3. Probabilidades de acierto y desactiero en las decisiones de inversiónde un gerente corporativo en una empresa enfocada y en una diveresificada

\begin{tabular}{ll}
\hline \multicolumn{1}{c}{ Empresa enfocada } & Empresa diversificada \\
\hline $\mathrm{P}\left(\mathrm{C}_{\mathrm{i}} / \mathrm{M}_{\mathrm{i}}\right)=\mathrm{P}\left(\mathrm{C}_{\mathrm{j}} / \mathrm{M}_{\mathrm{j}}\right)=\mathrm{P}\left(\mathrm{L}_{\mathrm{i}} / \mathrm{B}_{\mathrm{i}}\right)=\mathrm{P}\left(\mathrm{L}_{\mathrm{j}} / \mathrm{B}_{\mathrm{j}}\right)=\mathrm{q}$ & $\mathrm{P}\left(\mathrm{C}_{\mathrm{i}} / \mathrm{M}_{\mathrm{i}}\right)=\mathrm{P}\left(\mathrm{L}_{\mathrm{i}} / \mathrm{B}_{\mathrm{i}}\right)=\mathrm{q}$ \\
$\mathrm{P}\left(\mathrm{C}_{\mathrm{j}} / \mathrm{M}_{\mathrm{j}}\right)=\mathrm{P}\left(\mathrm{L}_{\mathrm{j}} / \mathrm{B}_{\mathrm{j}}\right)=\mathrm{r}$
\end{tabular}

Fuente: elaboración propia.

Por no ser relevante para el análisis de esta parte, omitiremos los factores $\phi \mathrm{y} k$. El factor $f$ del beneficio privado de los gerentes corporativos sí es relevante, debido a que si se hace un spin off este factor desaparece. Tal como lo realizó Stein (1997), se utiliza la regla de Bayes para calcular las probabilidades y el valor presente de la inversión para cada posible situación, dependiendo de si se trata de una corporación con diversificación o con enfoque. Los resultados se muestran en la tabla 4. 
Tabla 4. Probabilidades acierto en las decisiones de inversión de los gerentes corporativos para los estados de la naturaleza y valor presente de los flujos de caja de cada nivel de inversión, dependiendo de si la empresa es enfocada o diversificada

\begin{tabular}{|c|c|c|c|c|}
\hline \multirow[b]{2}{*}{$\begin{array}{l}\text { Estado } \\
\text { posible }\end{array}$} & \multicolumn{2}{|c|}{ Probabilidad } & \multicolumn{2}{|c|}{ Valor presente } \\
\hline & Enfoque & $\begin{array}{l}\text { Diversificación } \\
\text { no relacionada }\end{array}$ & Enfoque & $\begin{array}{c}\text { Spin off: inexistencia } \\
\text { de Gerencia } \\
\text { Corporativa }\end{array}$ \\
\hline BBLL & $\mathrm{p}^{2}\left(\mathrm{q}^{2}+\alpha(1-\mathrm{q})^{2}\right)$ & $\mathrm{p}^{2} \mathrm{qr}$ & $2\left((1-f) \theta_{2} y_{2}-I_{2}\right)$ & \multirow{2}{*}{$\left(\theta_{2} y_{2}+\theta_{1} y_{1}\right)-\left(I_{2}+I_{1}\right)$} \\
\hline BBLC & $p^{2}\left(q(1-q)-\alpha(1-q)^{2}\right)$ & $\mathrm{p}^{2} \mathrm{q}(1-\mathrm{r})$ & $(1-f)\left(\theta_{2} y_{2}+\theta_{1} y_{1}\right)-\left(I_{2}+I_{1}\right)$ & \\
\hline $\mathrm{BBCL}$ & $p^{2}\left(q(1-q)-\alpha(1-q)^{2}\right)$ & $p^{2}(1-q) r$ & $(1-f)\left(\theta_{1} y_{1}+\theta_{2} y_{2}\right)-\left(I_{1}+I_{2}\right)$ & \multirow{2}{*}{$2\left(\theta_{1} y_{1}-I_{1}\right)$} \\
\hline $\mathrm{BBCC}$ & $\mathrm{p}^{2}\left(\mathrm{q}(1-\mathrm{q})^{2}(1+\alpha)\right.$ & $\mathrm{p}^{2}(1-\mathrm{q})(1-\mathrm{r})$ & $2\left((1-f) \theta_{1} y_{1}-I_{1}\right)$ & \\
\hline BMLL & $\mathrm{p}(1-\mathrm{p}) \mathrm{q}(1-\mathrm{q})$ & $p(1-q) q(1-r)$ & $\left(\theta_{2} y_{2}+y_{2}\right)-2 I_{2}$ & \multirow{2}{*}{$\left(\theta_{2} y_{2}+y_{1}\right)-\left(I_{2}+I_{1}\right)$} \\
\hline BMLC & $\mathrm{p}(1-\mathrm{p}) \mathrm{q}^{2}$ & $\mathrm{p}(1-\mathrm{q}) \mathrm{qr}$ & $(1-f)\left(\theta_{2} y_{2}+y_{1}\right)-\left(I_{2}+I_{1}\right)$ & \\
\hline $\mathrm{BMCL}$ & $p(1-p)(1-q)^{2}$ & $p(1-p)(1-q)(1-r)$ & $(1-f)\left(\theta_{1} y_{1}+y_{2}\right)-\left(I_{1}+I_{2}\right)$ & \multirow{2}{*}{$\left(\theta_{1} y_{1}+y_{1}\right)-2 I_{1}$} \\
\hline BMCC & $p(1-p) q(1-q)$ & $p(1-p)(1-q) r$ & $(1-f)\left(\theta_{1} y_{1}+y_{1}\right)-2 I_{1}$ & \\
\hline MBLL & $(1-p) p(1-q) q$ & $(1-p) p(1-q) r$ & $(1-f)\left(y_{2}+\theta_{2} y_{2}\right)-2 I_{2}$ & \multirow{2}{*}{$\left(y_{2}+\theta_{1} y_{1}\right)-\left(I_{2}+I_{1}\right)$} \\
\hline MBLC & $p(1-p)(1-q)^{2}$ & $(1-p) p(1-q)(1-r)$ & $(1-f)\left(y_{2}+\theta_{1} y_{1}\right)-\left(I_{2}+I_{1}\right)$ & \\
\hline $\mathrm{MBCL}$ & $(1-p) p q^{2}$ & $(1-p) p q r$ & $(1-f)\left(y_{1}+\theta_{2} y_{2}\right)-\left(I_{1}+I_{2}\right)$ & \multirow{2}{*}{$\left(y_{1}+\theta_{1} y_{1}\right)-2 I_{1}$} \\
\hline MBCC & $(1-p)(1-q)$ & $(1-p) p q(1-r)$ & $(1-f)\left(y_{1}+\theta_{1} y_{1}\right)-2 I_{1}$ & \\
\hline MMLL & $(1-p)^{2}(1-q)^{2}(1+\alpha)$ & $(1-p)^{2}(1-q)(1-r)$ & $(1-f) y_{2}-I_{2}$ & \multirow{2}{*}{$\left(y_{2}+y_{1}\right)-\left(I_{2}+I_{1}\right)$} \\
\hline MMLC & $(1-p)^{2}\left(q(1-q)-\alpha(1-q)^{2}\right)$ & $(1-p)^{2}(1-q) r$ & $(1-f)\left(y_{2}+y_{1}\right)-\left(I_{2}+I_{1}\right)$ & \\
\hline MMCL & $(1-p)^{2}\left(q(1-q)-\alpha(1-q)^{2}\right)$ & $(1-p)^{2} q(1-r)$ & $(1-f)\left(y_{2}+y_{1}\right)-\left(I_{2}+I_{1}\right)$ & \multirow{2}{*}{$2\left(y_{1}-I_{1}\right)$} \\
\hline MMCC & $(1-p)^{2}\left(q^{2}+\alpha(1-q)^{2}\right)$ & $(1-p)^{2 q r}$ & $2\left((1-f) y_{1}-I_{1}\right)$ & \\
\hline
\end{tabular}

Fuente: elaboración propia. 
La combinación BBLL puede entenderse como el caso en que el estado de la naturaleza fue bueno para ambas unidades de negocio y el gerente tomo la decisión de hacer una inversión alta en ambas. La combinación BMLC representa el caso en el cual el estado de la naturaleza fue bueno para la unidad $i$ y malo para la unidad $j$; el gerente tomó la decisión de hacer una inversión alta en la unidad $i$ y baja en la unidad $j$.Un ejemplo numérico del resultado anterior se presenta en la tabla 5, en donde se calculan las probabilidades que varían por la inclusión del parámetro $\alpha$ :

Tabla 5. Cálculo de las probabilidades de cada decisión de inversión de un gerente corporativo, que se ve afectada por el parámetro que indica alta relación entre las unidades de negocio de una empresa

\begin{tabular}{|c|c|c|c|}
\hline Pyq & Alfa & Alfa & Alfa \\
\hline 0,5 & 0,3 & 0,5 & 0,7 \\
\hline 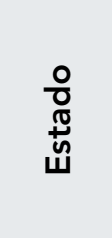 & $\begin{array}{l}\frac{0}{0} \\
\frac{\pi}{0} \\
\frac{.0}{\overline{0}} \\
\frac{0}{\pi} \\
0 \\
00 \\
0 \\
0\end{array}$ & $\begin{array}{l}\frac{0}{0} \\
\frac{\pi}{0} \\
\frac{.0}{\overline{0}} \\
\frac{0}{\pi} \\
00 \\
00 \\
0 \\
0\end{array}$ & $\begin{array}{l}\frac{0}{\pi} \\
\frac{\pi}{0} \\
\frac{.0}{\overline{0}} \\
\frac{0}{\pi} \\
0 \\
0 \\
0 \\
0\end{array}$ \\
\hline BBLL & 0,08125 & 0,09375 & 0,10625 \\
\hline BBLC & 0,04375 & 0,03125 & 0,01875 \\
\hline $\mathrm{BBCL}$ & 0,04375 & 0,03125 & 0,01875 \\
\hline $\mathrm{BBCC}$ & 0,08125 & 0,09375 & 0,10625 \\
\hline MMLL & 0,08125 & 0,09375 & 0,10625 \\
\hline MMLC & 0,04375 & 0,03125 & 0,01875 \\
\hline $\mathrm{MMCL}$ & 0,04375 & 0,03125 & 0,01875 \\
\hline MMCC & 0,08125 & 0,09375 & 0,10625 \\
\hline
\end{tabular}

Fuente: elaboración propia.

Se puede observar que, en la medida que $\alpha$ es mayor, la probabilidad de ocurrencia de las combinaciones BBLL, MMCC ${ }^{4}$ se incrementa, mientras que se disminuye la probabilidad de ocurrencia de combinaciones como BBLC y MMLC. ${ }^{5}$ Esto implica que cuando hay un alto grado de foco y la creencia del gerente corporativo es errada para una unidad

\footnotetext{
4 Probabilidades en las cuales se toma la misma decisión de inversión para las dos unidades de negocio.

5 Probabilidades en las cuales se toman decisiones de inversión diferentes para ambas unidades de negocio.
} 
de negocio, es más probable que sea errada para otra unidad de negocio y viceversa; sin embargo, si se suman las probabilidades de las combinaciones que producen valores presentes positivos (BBCC, BBLC, BBCL, BBLL, MMCC ${ }^{6}$ ) y se compara con la suma de las probabilidades que de las combinaciones que producen valores presentes negativos (MMLC, MMCL, MMLL), se puede ver que en la medida que aumenta $\alpha$, la suma de las primeras asciende a un mayor valor que la suma de las segundas. Esto quiere decir que la probabilidad de generar flujos de caja positivos para la empresa se incrementa cuando la empresa está enfocada.

Proposición 3. La decisión de una corporación para realizar un Spin-off de una unidad de negocio no relacionada, es directamente proporcional al nivel del parámetro alfa que indica el foco de las otras unidades de negocio de la corporación.

Prueba: la intuición detrás de esta proposición está basada en el hecho de que, en la medida que se hace diversificación no relacionada, $\alpha$ disminuye y por tanto se requiere un valor de $r$ mayor o igual a $q$, para obtener el mismo nivel de rentabilidad esperada. En una corporación con enfoque $q$ y $\alpha$ son altos, por tanto las combinaciones BBCC, BMLC, MBCL y MMCC ${ }^{7}$ tendrán una mayor probabilidad de ocurrencia; entonces, para incrementar el valor esperado, se necesitará un nivel de $r$ igual o mayor que $q$ para compensar el efecto de la diversificación. Lo anterior puede verse con un ejemplo numérico en la tabla 6.

6 Debe recordarse que MMCC no produce detrimento de valor, debido a que en ese caso hace una inversión $I_{1}$, que de todas formas genera valor para la corporación, al tener un valor presente positivo.

7 Dado que q es alta, entonces se incrementan las probabilidades en las cuales la gerencia corporativa acierta en la decisión de inversión. 
Tabla 6. Cálculo de la probabilidad de acierto en la decisión de inversión en una empresa diversificada ( $r$ ) que hace equivalente el valor presente de los flujos de caja de la inversión en una empresa enfocada, para las combinaciones que se imprementan por el incremento de $q$ y $\alpha$

\begin{tabular}{|c|c|c|c|c|c|c|}
\hline & $\mathrm{q}$ & & $Q$ & & $q$ & \\
\hline & 0,5 & & 0,7 & & 0,9 & \\
\hline $\mathrm{p}$ & alfa & & Alfa & & alfa & \\
\hline 0,5 & 0,3 & & 0,5 & & 0,7 & \\
\hline $\begin{array}{l}\frac{0}{0} \\
\frac{\pi}{ \pm 0} \\
\text { யூ }\end{array}$ & $\begin{array}{l}\frac{0}{0} \\
\frac{\pi}{0} \\
\frac{0}{\overline{0}} \\
\frac{0}{\pi} \\
\frac{0}{0} \\
0 \\
0 \\
0\end{array}$ & 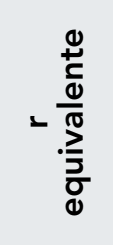 & 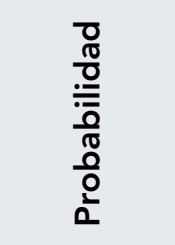 & 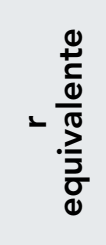 & 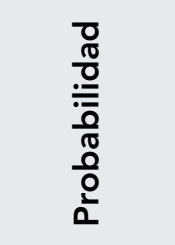 & 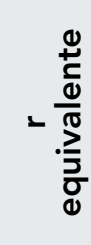 \\
\hline BBLL & 0,08125 & 0,65 & 0,13375 & 0,76 & 0,20425 & 0,91 \\
\hline BMLC & 0,0625 & 0,50 & 0,1225 & 0,70 & 0,2025 & 0,90 \\
\hline MBCL & 0,0625 & 0,50 & 0,1225 & 0,70 & 0,2025 & 0,90 \\
\hline MMCC & 0,08125 & 0,65 & 0,13375 & 0,76 & 0,20425 & 0,91 \\
\hline
\end{tabular}

Fuente: elaboración propia.

Se sabe que $r$ está en función inversa del número de unidades de negocio diferentes dentro de la corporación $(n)$, por tanto en una corporación con diversificación, $r$ será menor que $q$. Una forma de hacer $r$ igual o mayor que $q$ es disminuyendo $n$. Así, un incremento de $r$ se puede lograr cuando se separa la unidad de negocio y se configura como una empresa aparte; razón por la cual, los dueños de la corporación pueden tomar la decisión de hacer el spin off y conservar la propiedad de la nueva empresa, con el propósito de aumentar el valor presente neto esperado de la inversión. La argumentación anterior demuestra la validez de la proposición. La intuición desarrollada en este numeral complementa las ideas de Williamson $(1975)^{8}$ y Daley, Mehrotra y Sivakumar (1997). ${ }^{9}$

8 Williamson (1975) indica que si bien los gerentes corporativos están mejor informados acerca de las buenas oportunidades de inversión, en la medida que la diversificación se incrementa surgen los problemas por el aumento en la complejidad de la supervisión y la dificultad de tomar buenas decisiones acerca de la asignación de recursos.

9 Daley, Mehrotra y Sivakumar (1997) indican que más allá de la ganancia en eficiencia operativa, los spin off crean valor porque permiten remover unidades de negocio no relacionadas y permiten a los gerentes enfocar su atención. 
Se debe anotar que el incremento de $r$, a través del spin off debe compensar la aparición de las restricciones de capital, pero tiene a su favor el hecho de que no se requiere ahora incurrir en el costo del beneficio privado del gerente corporativo. En la tabla 7 se muestra el cálculo de $r$ ( $r$ de umbral) a partir del cual la rentabilidad del spin off supera la rentabilidad de no hacerlo para las combinaciones (BBCC, BBLC, BBCL, BBLL, MMCC), que se incrementan con la estrategia de enfoque.

Tabla 7. Cálculo de la probabilidad de acierto en la decisión de inversión en una empresa diversificada (r) umbral que no compensa la pérdida de enfoque y la desaparición del beneficio privado del gerente corporativo

\begin{tabular}{|c|c|c|c|c|c|}
\hline $\mathrm{I}_{1}$ & 1 & $\theta_{1}$ & 1,1 & $f$ & \multirow{5}{*}{$\begin{array}{c}r \\
\text { umbral }\end{array}$} \\
\hline $\mathrm{I}_{2}$ & 2 & $\theta_{2}$ & 1,4 & 0,05 & \\
\hline $\mathrm{Y}_{1}$ & 1,1 & $\mathrm{Q}$ & $\mathrm{P}$ & alfa & \\
\hline $\mathrm{Y}_{2}$ & 1,8 & 0,6 & 0,5 & 0,3 & \\
\hline 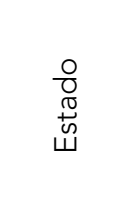 & $\begin{array}{l}\overline{0} \\
\frac{\pi}{0} \\
\overline{\underline{\bar{O}}} \\
\overline{0} \\
0 \\
0 \\
0 \\
0\end{array}$ & 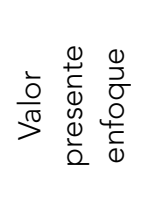 & 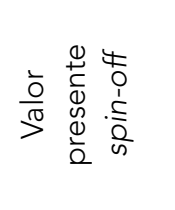 & 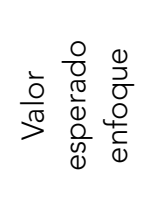 & \\
\hline BBLL & 0,1020 & 0,7880 & 0,7300 & 0,0804 & 0,7340 \\
\hline BBLC & 0,0480 & 0,5435 & 0,7300 & 0,0261 & 0,7618 \\
\hline $\mathrm{BBCL}$ & 0,0480 & 0,5435 & 0,4200 & 0,0261 & 0,6211 \\
\hline $\mathrm{BBCC}$ & 0,0520 & 0,3990 & 0,4200 & 0,0207 & 0,5060 \\
\hline MMCC & 0,1020 & 0,0900 & 0,2000 & 0,0092 & 0,3060 \\
\hline
\end{tabular}

Fuente: elaboración propia.

Excepto en los casos de MMCC y BBCC, ${ }^{10} r$ es superior a $q$. Quiere decir que una corporación que hizo diversificación no relacionada, tomará la decisión de hacer un spin-off, cuando piense que el incremento en la probabilidad de acertar las decisiones de inversión en la unidad de negocio no relacionada, se incrementará lo suficiente para igualar al menos, la probabilidad asociada con la rentabilidad de tener unidades de negocio relacionadas

10 En estos casos (r) es menor, debido a la desaparición del beneficio privado del gerente corporativo y a que la decisión de hacer una inversión baja en ambas unidades de negocio, dada la señal, sería la misma inversión que se hace en el caso del spin off, cuando se enfrentan restricciones de capital. Sin embargo, es de anotarse que si el spin off hace que (r) se incremente, el umbral requerido sería más fácilmente superado y se obtendría igualmente una rentabilidad esperada mayor, por lo cual al hacer spin off se hace una estrategia dominante. 
y compensar las posibles restricciones de capital. Además esta decisión será impulsada porque se sabe que la disminución del beneficio privado del gerente corporativo incrementará los incentivos del gerente de la unidad de negocio para mejorar su desempeño.

\section{El grupo empresarial Bavaria: su proceso de diversificación y de spin off}

En este parte se utiliza el caso del grupo empresarial Bavaria para contrastar las predicciones que el análisis teórico arrojó en el numeral anterior. Inicialmente, se hace una descripción histórica del grupo, para después centrarnos en evidenciar que la gerencia corporativa de este grupo disponía de un amplio mercado interno de capital generado por unidades de negocio que poseían alta liquidez y que ese mercado interno de capital contribuyó al proceso de diversificación del grupo. Además, daremos cuenta de que el proceso de diversificación de Bavaria implicó que dentro de su estructura corporativa se combinaran unidades de negocio con alta y baja relación entre ellas y finalmente, que el grupo realizó un spin off agrupando por un lado las empresas relacionadas con la producción y comercialización de bebidas, y por otro lado, bajo una gerencia corporativa distinta, el resto de empresas no relacionadas.

\subsection{El desarrollo histórico del grupo empresarial Bavaria}

Desde su creación en 1889, Bavaria empezó su integración vertical mediante la producción directa o indirecta de cebada, la fabricación de botellas con la Vidriera Fenicia y la adquisición de la mina de carbón de Zipacón. Hacia 1930 se presentó una importante transformación organizativa de la empresa, al convertirse en Consorcio de Cervecería Bavaria S.A., proceso de concentración y centralización del capital producto de la unión de varias empresas. En 1934 el consorcio contaba con ocho plantas cerveceras distribuidas en siete ciudades, que cubrían estratégicamente el centro y el norte del mercado interno colombiano. ${ }^{11}$

A partir de años 60 y en adelante, el consorcio cervecero aceleró su proceso de integración, al mismo tiempo que se diversificó hacia otras actividades económicas, como la

11 Adaptado de http://www.grupobavaria.com/espanol/queesbavaria/historia.php 
metalmecánica, la financiera y la inmobiliaria. En esta década se instauró como uno de los conglomerados más poderosos del país, con un conjunto de unidades de producción y de distribución organizado en varias empresas, bajo nombres diferentes, pero obedeciendo a un centro de decisión único. En 1983 se inició la expansión internacional de Bavaria con la adquisición de un holding panameño llamado Ladco (Latin Developement Corporation). A través de esta adquisición, Bavaria se hizo al control de casi todo el mercado cervecero ecuatoriano y en Colombia obtuvo el 29 \% de la propiedad en Cervunión. ${ }^{12}$ Desde la década de los 80 la expansión del Grupo también se orientó hacia las de servicios de empleo temporal y en 1984 fundó Colenvases, empresa encargada de la producción de latas de aluminio para envasar cerveza y gaseosas en recipientes no retornables. En 1985 se creó Impresora del Sur en la ciudad de Cali, empresa encargada de la producción de etiquetas y afiches para el mercado cervecero y la papelería impresa necesaria para la organización. En 1987, 92,2 \% de las inversiones del grupo Bavaria estaban representadas en los títulos de propiedad de 48 empresas de diferentes sectores (Sanabria, 2003).

El 31 de octubre de 1997 fue aprobado un proyecto de spin off. De conformidad con esta medida, el Grupo Empresarial Bavaria se separó en dos sociedades anónimas diferentes: la primera continuó llamándose Bavaria S.A., dedicada a la producción, venta de cervezas y relacionados, y la segunda se denominó Valores Bavaria S.A., dedicada exclusivamente a las actividades de inversión. Actualmente, Bavaria S.A. es una empresa insigne y líder para la economía colombiana, un actor de primera línea en Latinoamérica y un fuerte competidor en el mercado cervecero mundial. A partir del año 2004, Bavaria logró ocupar el primer lugar en la industria cervecera de Colombia, Ecuador, Panamá y Perú, y es la segunda compañía cervecera más grande de Sudamérica y la décima del mundo. En 2005 fue adquirida por el grupo Sab Miller, quien le ha dado un mayor impulso obteniendo resultados importantes. La tabla 8 muestra las principales cifras de Bavaria y la tabla 9 muestra los volúmenes de ventas para el cierre de 2007.

12 Adaptado de Investigación de Origen y Estructura del Grupo Santo Domingo, Material resultado de investigación con el apoyo de la Facultad de Ciencias Económicas de la Universidad Nacional de Colombia y de la División de Investigaciones de Bogotá, coordinado por el profesor Bernardo Parra Restrepo y disponible en

http://www.virtual.unal.edu.co/cursos/economicas/2006930/. 
Tabla 8. Principales cifras de Bavaria a diciembre de 2007

\begin{tabular}{lr}
\hline \multicolumn{2}{c}{ Valores en millones de pesos } \\
\hline Ventas & \multicolumn{1}{c}{012,395} \\
\hline Utilidad operativa & $1,294,463$ \\
\hline Ebitda & $1,734,502$ \\
\hline Utilidad neta & 694,603 \\
\hline Activos totales & $9,450,836$ \\
\hline Propiedad planta y equipo & $2,265,841$ \\
\hline Pasivo Total & $5,269,364$ \\
\hline Deuda Financiera & $3,069,408$ \\
\hline Patrimonio & $4,146,622$ \\
\hline
\end{tabular}

Fuente: Informe anual de Bavaria (2007).

Tabla 9. Ventas de Bavaria en volúmenes de hectolitros

\begin{tabular}{lrrrr}
\hline \multicolumn{1}{c}{ Categoría } & $\mathbf{2 0 0 7}$ & $\mathbf{2 0 0 6}$ & \multicolumn{2}{c}{ Variación } \\
\hline Cervezas & 17.647 .105 & 15.216 .543 & 2.430 .562 & $16.0 \%$ \\
\hline Maltas & 2.113 .994 & 1.942 .609 & 171.385 & $8.8 \%$ \\
\hline Refrescos de fruta $\left(^{*}\right)$ & 218.286 & 535.287 & $(317.001)$ & $(59.2 \%)$ \\
\hline Aguas & 2.286 .309 & 2.308 .301 & $(93.992)$ & $(3.9 \%)$ \\
\hline Total volumen de ventas & $\mathbf{2 2 . 2 6 5 . 6 9 4}$ & $\mathbf{2 0 . 0 7 4 . 7 4 0}$ & $\mathbf{2 . 1 9 0 . 9 5 4}$ & $\mathbf{1 0 . 9 \%}$ \\
\hline
\end{tabular}

(*) Hls vendidos hasta el 31 de mayo del 2007.

http://www.grupobavaria.com/pdfs/esp/informeanual07.pdf

Fuente: Informe anual de Bavaria (2007).

\subsection{La existencia de un mercado interno de capitales en Bavaria y su utilización para la realización del proceso de diversificación}

En este apartado se enumeran los hechos que evidencian la existencia de un mercado interno de capitales en el grupo Bavaria y su utilización para la diversificación no relacionada. Primero, Bavaria actuaba como casa matriz o núcleo que poseía participaciones en otras 140 compañías. El presidente de Bavaria tenía también el control de las otras empresas subordinadas y estaba encargado de la orientación general y el seguimiento 
de los gerentes de cada empresa. Era tan evidente la unidad de mando, que en 1992 el sindicato de Bavaria logró que el Ministro de Trabajo de esa época declarara la unidad de empresa (Sanabria, 2003). Segundo, existía una disponibilidad interna de capital proporcionada por las cervecerías que generaban una alta liquidez. La dinámica operacional del negocio de la cerveza hace que los recaudos por ventas se hagan en el corto plazo, mientras que los pagos de impuestos y proveedores se hacen en plazos mayores, lo que permite mantener una alta liquidez de manera permanente, que posibilita la existencia del mercado interno de capital. Una evidencia de lo anterior son los niveles de productividad de capital de trabajo mostrados en la figura 3.

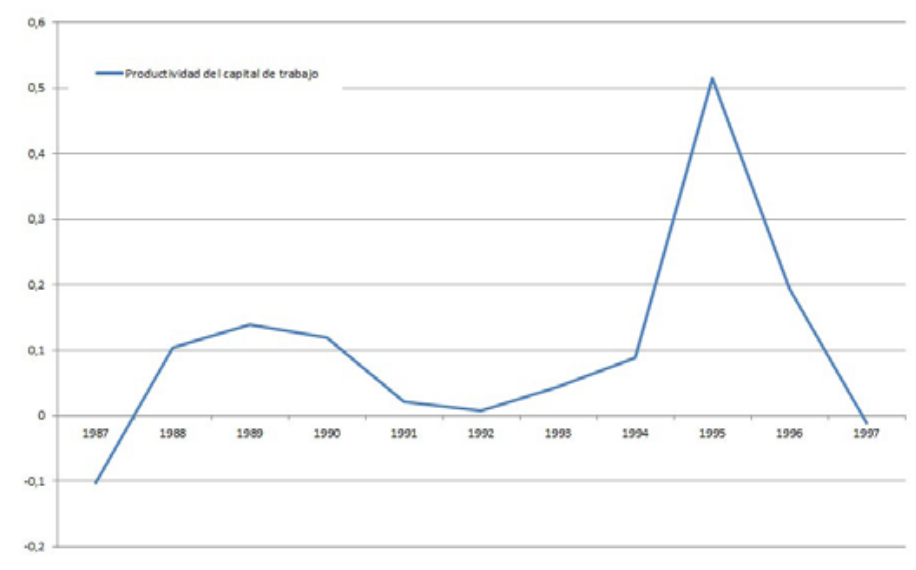

Figura 3. Productividad del capital de trabajo neto operativo en Bavaria del año 1987 a 1997 Fuente: Cálculos hechos por el autor con base en datos tomados de Sanabria (2003).

Si analizamos un período de 10 años antes del Spin-off (1987-1997) se denota que en algunos años Bavaria trabajaba con capital de trabajo negativo (1987 y 1997) y en los años 19991 a 1994, requirió menos de 9 centavos de capital de trabajo por peso vendido. La liquidez de Bavaria puede corroborarse en el gráfica 2, se muestra una comparación entre el efectivo generado por la operación y el acumulado de sus destinaciones (Servicio de la deuda, dividendos e inversiones en propiedad planta y equipo). Como puede notarse, excepto para el año 1995, el efectivo generado por la operación supera ampliamente las destinaciones, lo cual establece la posibilidad de generación del mercado interno de capital. 


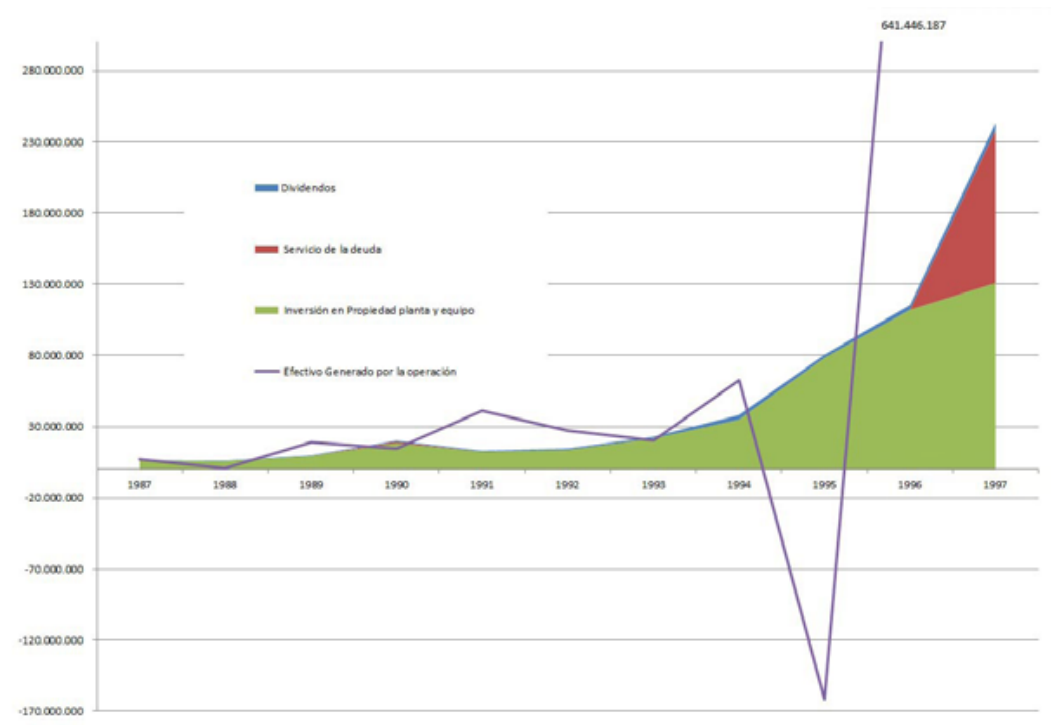

Figura 4. Operación y destinación del efectivo generados por la operación en Bavaria del año 1987 a 1997

Fuente: Cálculos hechos por el autor con base en datos tomados de Sanabria (2003).

Tercero, Bavaria emprendió un proceso de diversificación desde los inicios de su historia, que se fue tornando más agresivo en la medida en que el grupo empresarial evolucionó. ${ }^{13}$ Se puede evidenciar que la gerencia corporativa de Bavaria utilizó estos excesos de caja en el proceso de diversificación de Bavaria, ya que con ellos se apalancaron no solo la adquisición de las otras unidades de negocio, sino el financiamiento de los proyectos de las unidades de negocio adquiridas. Por ejemplo, en los documentos de la banca de inversión que ayudó a estructurar el proceso de adquisición de la Unión de Cervecería Peruanas Backus y Johnston (Backus) en Perú (la Internacional Finance Corporation) se menciona que para cuando Bavaria y Valores Bavaria concluyen el proceso de escisión acordaron realizar una capitalización. Los créditos otorgados por Bavaria se convirtieron en acciones de Valores Bavaria a favor de los accionistas de Bavaria, lo cual denota la existencia de múltiples transferencias de recursos de Bavaria a sus subordinadas para financiar sus operaciones, es decir, se usa un mercado interno de capital para financiar

13 En Origen y Estructura del Grupo Santo Domingo, material resultado de investigación con el apoyo de la Facultad de Ciencias Económicas de la Universidad Nacional de Colombia y de la División de Investigaciones de Bogotá, coordinado por el profesor Bernardo Parra Restrepo y disponible en http://www.virtual.unal.edu.co/cursos/economicas/2006930/; se muestra una lista de las empresas a nivel nacional e internacional que controlaba el grupo Bavaria en el momento del Spin-off. En total son 140 empresas, 29 relacionadas con la producción de cerveza, 13 de servicios generales, 11 del sector financiero, 8 del sector de transporte, 9 del sector de la construcción e inmobiliario, 11 del sector de las telecomunicaciones, 31 medios de comunicación, 4 de la industria automotriz, 4 del sector salud, 12 compañías de inversiones generales y 8 del sector agropecuario. 
la diversificación (Bernal \& Lubrano, 2007). Ramirez, Jimenes y Garrido (2003) publican un aparte de una entrevista con fuentes de Bavaria, en donde los ejecutivos afirman que han financiado la expansión del grupo con una mezcla de Financiación interna (mercado interno de capital) y financiación nacional e internacional. Parra (2004) concluyó que las cervecerías eran buenas "vacas lecheras" ${ }^{14}$ y que los fondos provenientes de las cerveceras utilizados para financiar otras empresas del grupo en el año 2001, fecha de terminación del spin off, ascendían a varios millones de pesos. Con lo anterior queda claro que para el caso de Bavaria usaron las unidades de negocio con excesos de liquidez, para diversificarse de manera no relacionada, porque la existencia de un mercado interno de capital permitió derribar las restricciones de financiación para incrementar su rentabilidad corporativa.

\subsection{La realización del spin off: Bavaria S.A. y Valores Bavaria S.A.}

En 1997 Bavaria detiene la diversificación y empieza una estrategia de focalización. Tratando de concentrar los objetos de los diferentes tipos de negocio, inicia su primer spin off, mediante el cual pretende la separación de las empresas de cervezas y bebidas que continúan bajo el nombre de Bavaria S.A. y las demás empresas son separadas y conforman una compañía independiente llamada Valores Bavaria S.A. Esta separación obligó a la presentación de estados financieros separados, quedando las dos compañías tal como se muestra en la tabla 10.

Tabla 10. Estados financieros en fecha de escisión de 1997 en millones de pesos

\begin{tabular}{llll}
\hline \multicolumn{1}{c}{ Concepto } & \multicolumn{1}{c}{$\begin{array}{c}\text { Grupo empresarial } \\
\text { Bavaria S.A. }\end{array}$} & Bavaria S.A. & Bavaria S.A. \\
\hline Activos & 4906220 & 3420777 & 1485443 \\
\hline Pasivos & 1025790 & 856977 & 1316630 \\
\hline Patrimonio & 3880430 & 2563800 & 1000 \\
\hline Capital autorizado & 1000 & 1000 & 701 \\
\hline Capital Suscrito y Pagado & 768 & 768 & \\
\hline
\end{tabular}

Fuente: Sanabria (2003).

14 Refiriéndose a su alta liquidez. 
Este primer intento de separación fue más nominal y legal que real, por cuanto la nueva compañía Valores Bavaria continuaría gerenciada por el equipo administrativo y gerencial de Bavaria S.A., y también utilizaría los mismos recursos físicos y operativos de Bavaria S.A. De igual manera, se siguió haciendo uso del mercado interno de capitales, puesto que Bavaria S.A. realizó préstamos a Valores Bavaria en 1999 para inversiones en la compañía de telefonía móvil celumóvil (hoy Telefónica); Avianca (aerolínea); Comunican (diario $E l$ Espectador); Cofinorte (empresa financiera); Caracol Televisión, Parque Central Bavaria (inmobiliaria); Inversiones Cromos S.A (editora de revistas), así como para otras veinte empresas. ${ }^{15}$ Sin embargo, los resultados de valores Bavaria no eran buenos y en el periodo 2000-2001 las pérdidas ascendieron a cerca de \$1 billón de pesos. En ese momento es nombrado como presidente del grupo el español Javier Aguirre, quien propone comprometerse con un esquema de transparencia en la información, que genere confianza en los mercados con le objetivo de convertir a Valores Bavaria en un holding internacional. Sin embargo, los analistas dicen que Julio Mario Santo Domingo se resiste a reducir el tamaño del grupo y, a la hora de tomar decisiones definitivas, no les da a los ejecutivos la autonomía que necesitan. Esto explicaría por qué tardan tanto las soluciones para las empresas del grupo. ${ }^{16}$ Las opiniones de los analistas para ese entonces son consistentes con el análisis teórico realizado para la proposición 3. Es evidente que cuando se maneja un grupo con 140 empresas de 11 sectores diferentes estamos ante un caso de alta diversificación y por ende disminuye la probabilidad $(r)$ de acierto en las decisiones de inversión de una gerencia corporativa. También es evidente que el objetivo de Bavaria al realizar el spin off de las unidades de negocio no relacionadas fue independizar administrativa y financieramente a Bavaria S.A. de Valores Bavaria S.A. y lograr que cada compañía se enfocara por completo en su actividad principal (Bernal \& Lubrano, 2007), lo cual confirma que al existir un alfa alto $(\alpha)$ por el foco de las unidades de negocio relacionadas con la producción de cerveza, se incrementa la propensión a la realización del spin off.

El proceso de separación continuó su marcha y culminó con un segundo spin off en 2001, quedando agrupado todo el negocio cervecero y de bebidas dentro del Grupo Empresarial Bavaria (GEB). Los créditos otorgados por GEB se convirtieron en acciones de Valores Bavaria, a favor de los accionistas de Bavaria. Con este segundo spin off se abrió

15 Para más información visitar http://www.finanzas.com/id.3003051/noticias/noticia.htm

16 Valores Bavaria se Juega su futuro. Revista Dinero. Bogotá. Colombia. 03/22/2002. http://WWW.DINERO.COM/WF_INFOARTICULO.ASPX?IDART=3105 
la independencia administrativa y financiera entre Bavaria S.A. y Valores Bavaria S.A., y se hizo que cada empresa trabajara con sus propios flujos de caja y se enfocara por completo en su actividad principal.

A su vez, esta operación permitió obtener una mayor transparencia en las cuentas del grupo, lo que permitió, además, realizar ventas y alianzas estratégicas en el campo de la actividad cervecera (con Sab Miller finalmente) y facilitar la venta de negocios a algunos inversionistas extranjeros (como Caracol a Prisa, Avianca a Sinergy y Celumovil a Bellsouth), con el propósito de incrementar la probabilidad de acierto en las decisiones de inversión. Las menciones de los analistas acerca de la demora en la toma de decisiones y el pobre desempeño de las empresas con baja relación, agrupadas en Valores Bavaria, podría ser también un indicio del fenómeno de dilución de esfuerzo mencionado en la proposición 2 del análisis teórico. Este punto requiere mayor comprobación, ya que se debe establecer si las firmas con alta especialización dentro de la corporación eran las de peor desempeño en el grupo; también se debe analizar el desempeño de la empresa mientras era parte del grupo y después de que salió de él, controlando si realmente su desempeño mejoró debido al desempeño gerencial, es decir, por una disminución en la dilución de esfuerzo. También podría compararse el desempeño de las empresas del grupo con otras del mismo sector, tamaño y ubicación similar, que no hagan parte de una unidad corporativa, pero esto quedará para un próximo trabajo.

\section{Conclusiones}

Las empresas enfrentan restricciones de financiación cuando no tienen acceso a mercados internos de capital, porque para el proveedor de capital siempre será una estrategia dominante restringir la financiación que otorga. Como un gerente siempre querrá controlar un mayor número de recursos e incrementar la rentabilidad corporativa, cuando hay mercados internos de capital la estrategia dominante del gerente corporativo será hacer inversiones grandes en nuevas unidades de negocio que considera rentables, para incrementar la rentabilidad global de la empresa. Luego, las empresas con unidades de negocio caracterizadas por excesos de liquidez, se diversifican, porque la existencia de un mercado interno de capital permite derribar las restricciones de financiación para incrementar su rentabilidad corporativa; lo anterior quedó evidenciado en el caso del Grupo Empresarial Bavaria. 
De otra parte, en una corporación con varias unidades de negocio relacionadas, la probabilidad de que la gerencia corporativa acierte en una decisión de inversión es alta. Cuando la corporación decide incrementar la diversificación, incorporando unidades de negocio no relacionadas, la probabilidad de acierto en las decisiones de inversión en estas últimas es menor; ee sabe que la probabilidad de acierto en las decisiones de inversión está en función inversa del número de unidades de negocio diferentes en la corporación. Por tanto, existen niveles de diversificación que pueden reducir el valor esperado de la rentabilidad corporativa. Cuando una corporación llega a estos niveles, si desea incrementar nuevamente la rentabilidad esperada, se requiere que la probabilidad de acierto en las decisiones de inversión en las unidades no relacionadas se incremente, en cuyo caso el spin off se convierte en una estrategia acertada. Entonces, la decisión de una corporación para realizar un spin off de una unidad de negocio no relacionada es directamente proporcional al nivel de foco de las otras unidades de negocio de la corporación. Tal fue el caso de Bavaria, en donde los niveles de relación entre las unidades de negocio cerveceras y el alto grado de diversificación hizo indispensable el spin off, incluso muy a pesar de sus dueños. Está podría ser una razón por la cual en algunos grupos económicos se observan ciclos de diversificación y ciclos de enfoque. Cuando el grupo económico desea incrementar la rentabilidad inicia su proceso de diversificación, pero una vez llega al umbral que hace que la probabilidad de acierto en sus decisiones de inversión se reduzca, empieza nuevamente su proceso de enfoque, realizando separación de sus unidades no relacionadas. Y el ciclo comienza nuevamente.

Finalmente, mencionamos que la diversificación puede beneficiar a los gerentes, ya que puede otorgarles poder, prestigio y una mayor remuneración asociada con el gerenciamiento de una firma grande; sin embargo, cuando la dilución del esfuerzo es muy alta, los flujos de caja caen y la probabilidad de malos resultados se incrementa. De este modo, habrá más dilución de esfuerzo cuando el gerente de la unidad de negocio siente que la gerencia corporativa no aporta nada a su trabajo, pero si extrae beneficios; si la calidad del aporte del gerente corporativo es menor cuando existe alta diversificación, entonces habrá más dilución de esfuerzo cuando los niveles de diversificación son altos. 


\section{Referencias}

Aggarwal, R., \& Samwick, A. (2003). Why Do Managers Diversify Their Firms? Agency Reconsidered, The Journal of Finance, 58(1), 71-118.

Ahn, S., \& Denis, D. (2004). Internal Capital Markets and Investment Policy: Evidence from Corporate Spinoffs, Journal of Financial Economics, 71, 489-516.

Amihud, Y., \& Lev, B. (1981). Risk Reduction as a Managerial Motive for Conglomerate Mergers, Bell Journal of Economics, 12, 605-617.

Aron, D. (1991). Using the Capital Market as a Monitor: Corporate Spinoffs in an Agency Framework, RAND Journal of Economics, 22(4), 505-518.

Berger, P., \& Ofek, E. (1995). Diversification's effect on firm value. Journal of Financial Economics, 37, 39-65.

Bernal, A., \& Lubrano, M. (2007). Crecimiento, Financiamiento y Gobierno Corporativo: El Proceso de Crecimiento de Bavaria, Una Empresa con Vocación Global. Pensilvania: International Finance Corporation.

Brush, T. (1996) Predicted Change in Operational Synergy and Post-Acquisition Performance of Acquired Businesses. Strategic Management Journal, 17, 1-24.

Campa, J., \& Kedia, S. (2002). Explaining the diversification discount.Journal of Finance, 57 , 1931-1962.

Chandler, A. (1977). The Visible Hand. Cambridge: Belknap Press.

Daley, L., Mehrotra, V., \& Sivakumar, R. (1997) Corporate Focus and Value Creation: Evidence from Spinoffs. Journal of Financial Economics, 45, 257-281.

Gertner, R., Scharfstein, D., \& Stein, J. (1994). Internal Versus External Capital Markets. The Quarterly Journal of Economics, 109(4), 1211-1230.

Gertner, R., Powers, E., \& Scharfstein, D. (2002). Learning about Internal Capital Markets from Corporate Spin-offs. The Journal of Finance, 57(6), 2479-2506.

Grossman, S., \& Hart, O. (1986). The Costs and Benefits of Ownership: A Theory of Vertical and Lateral Integration. Journal of Political Economy, 94, 691-719.

Hart, O., \& Moore, J. (1990), Property Rights and the Nature of the Firm. Journal of Political Economy, 98, 1119-1158.

Hermalin, B., \& Weisbach, M. (1998). Endogenously Chosen Boards of Directors and Their Monitoring of the CEO. American Economic Review, 88, 96-118.

Hite, G., \& Owers, J. (1983). Security Price Reactions Around Corporate Spin-off Announcements. Journal of Financial Economics, 12, 409-436. 
Hoechle, D., Schmid, M., Walter, I., \& Yermack, D. (2012). How Much of the Diversification Discount Can Be Explained by Poor Corporate Governance? Journal of Financial Economics, 103, 41-60.

Jensen, M. (1986). Agency Costs of Free Cash Flow, Corporate Finance and Takeovers. American Economic Review, 76, 323-329.

Jensen, M., \& Murphy. K. (1990). Performance Pay and Top Management Incentives. Journal of Political Economy, 98(2), 225-264.

Khanna, T., \& Palepu, K. (2000). Is Group Affiliation Profitable in Emerging Markets? An Analysis of Diversified indian Business Groups. Journal of Finance, 55, 867-891.

Kudla. R., \& Mclnish, T. (1983). Valuation Consequences of Corporate Spinoffs. Review of Business and Economic Research, 9, 71-77.

Lamont, O. (1997). Cash Flaw and Investment: Evidence from Internal Capital Markets. Journal of Finance, 52, 83-110.

Lang, L., \& Stulz, R. (1994). Tobin's q, Corporate Diversification and Firm performance. Journal of Political Economy, 102, 1248-1280.

Lewellen, W. (1971). A Pure Financial Rationale for the Conglomerate Merger.Journal of Finance, 26, 521-537.

Lins, K., \& Servaes, H. (2002). Is Corporate Diversification Beneficial in Emerging Markets. Financial Management, 31, 5-31.

Manne, H. (1965). Mergers and Market for Corporate Control. Journal of Political Economy, $73,110-120$.

Mansi, S., \& Reeb, D. (2002). Corporate Diversification: What Gets Discounted? The Journal of Finance, 57(5), 2167-2183.

Meyers, M., Milgron, P., \& Roberts, J. (1992) Organizational Prospects, Influence Cost and Ownership. Journal of Economics and Management Strategy, 1, 9-35.

Miles, J., \& Rosenfeld, J. (1983). An Empirical Analysis of the Effects of Spin-off Announcements on Shareholder Wealth. Journal of Finance, 38, 1597-1606.

Morck, R., Schleifer, A., \& Vishny, R. (1990). Do managerial Objectives Drive Bad Acquisitions, The Journal of Finance, 45, 31-48.

Parra, B. (2004), Investigación de origen y estructura del grupo Santo Domingo, material resultado de investigación con el apoyo de la Facultad de Ciencias Económicas de la Universidad Nacional de Colombia y de la División de Investigaciones de Bogotá. Recuperado de http://www.virtual.unal.edu.co/cursos/economicas/2006930/ 
Rajan, R., Servaes, H., \& Zingales, L. (2000). The Cost of Diversity: The Diversification Discount and Inefficient Investment". Journal of Finance, 55(1), 35-80.

Ramaswamy, K., Purkayastha, S., \& Petitt, B. (2017). How do Institutional Transitions Impact the Efficacy of Related and Unrelated Diversification Strategies Used by Business Groups? Journal of Business Research, 72, 1-13.

Ramírez C., Jiménez, A., \& Garrido, J. (2003), La inversión colombiana en el exterior: Bavaria y su estrategia internacional con las cervezas. Estudios Gerenciales, Universidad Icesi, 1, 57-78.

Sanabria, R. (2003). Bavaria y Valores Bavaria S. A. Monografias de Administración, Serie Casos, Universidad de los Andes. Bogotá.

Scharfstein, D., \& J. Stein (2000). The Dark Side of Internal Capital Markets: Divisional Rent Seeking and Inefficient investments. Journal of Finance, 55, 2537-2564.

Schipper, K., \& Smith, A. (1983). Effects of Recontracting on Shareholder Wealth: The Case of Voluntary Spin-offs. Journal of Financial Economics, 12, 437-467.

Shin, H., \& Stulz, R. (1998), Are Internal Capital Markets Efficient? The Quarterly Journal of Economics, 113(2), 531-552.

Shleifer, A., \& Vishny, R. (1989). Managerial Entrenchment: The Case of Manager Specific Investments. Journal of Financial Economics, 25, 123-139.

Stein, J. (2003). Agency, Information and Corporate Investments. En G. Constantides, M. Harris and L. Stulz (Eds.), Handbook of the Economics of Finance (pp.121 -183). Ámsterdam.

Stultz, R. (1990). Managerial Discretion and Optimal Financing Policies. Journal of Financial Economics, 26, 3-27.

Stein, J. (1997) Internal Capital Markets and the Competition for Corporate Resources. The Journal of Finance, 52, 111-133.

Teece, D. (1982). Toward an Economic Theory of the Multiproduct Firm. Journal of Economic Behavior and Organization, 99(3), 39-63.

Weston, F. (1970). The Nature and Significance of Conglomerate Firms. St. John's Law Review, 44, 66-80.

Williamson, O. (1975). Markets and Hierarchies: Analysis and Antitrust Implications. New York: The Free Press.

Zheng, S. (2017). Can Corporate Diversification Induce More Tax Avoidance? Journal of Multinational Financial Management, 41,47-60. 